\title{
Interdroplet distance in a structured monolayer of liquid microdroplets levitating over hot liquid surface
}

\author{
Almira Shatekova ${ }^{1,2, *}$ \\ ${ }^{1}$ Kutateladze Institute Institute of Thermophysics, 630090 Novosibirsk, Russia \\ ${ }^{2}$ Novosibirsk State University, 630090 Novosibirsk, Russia
}

\begin{abstract}
Structured arrays of liquid microdroplets levitating over a heated liquid film have been observed in experimental works, but the nature of this phenomena is not completely understood. The aim of this work is to measure the distance between drops and the size of monolayer depending on the main parameters. We found that the average distance between drops increases with time from 40 to $100 \mu \mathrm{m}$. And the size of monolayer increases with time from approximately $1 \mathrm{~mm}$ to $5 \mathrm{~mm}$. The distance between drops increases when approaching the boundary of monolayer.
\end{abstract}

\section{Introduction}

It is known that an ordered "monolayer" structure can be formed over a heated liquid surface $[1,2]$. A monolayer is a highly ordered triangular structure consisting of hundreds and thousands microdroplets (Fig.1).

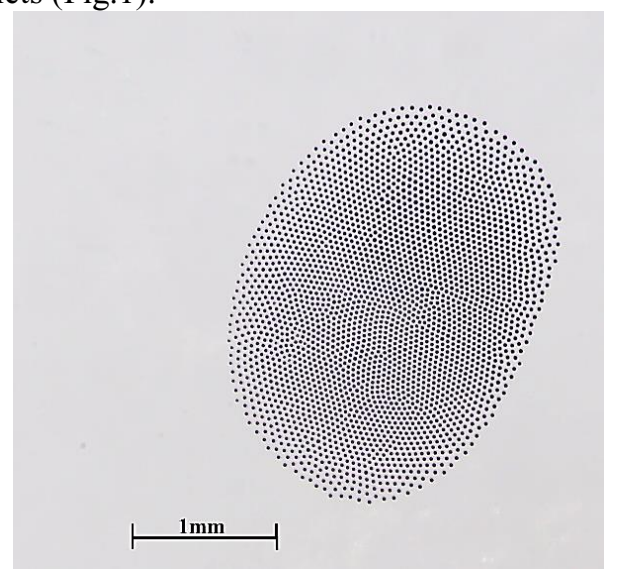

Fig. 1. The monolayer (top view). The thickness of liquid film $\mathrm{h}=0.5 \mathrm{~mm}$ and temperature of substrate $\mathrm{T}=83^{\circ} \mathrm{C}$.

\footnotetext{
* Corresponding author: shatekova@list.ru
} 
The microdroplets are levitating above the liquid surface at a distance comparable to the droplet diameter. The typical size of the microdroplets in a monolayer varies from 10-100 $\mu \mathrm{m}$. The study of monolayer can be used for practical applications, for example, spray cooling, when a steam of droplets is directed to the heated surface, it is important to know under what conditions the microdroplets levitate [3]. It is also important to know conditions of levitating for delivering drugs via sprays [4]. Moreover, microdroplets can be used as tracers. On trajectories, it is possible to restore the microscale gas-vapor flows near the liquid-gas contact line [5,6]. Initially the levitating microdroplets are drops of condensate arising from the evaporation of the heated liquid. After drops gain enough mass and then fall down on the different parts of the liquid surface (supported by the steam originated at the surface of liquid, as discussed in [2]) and collected in an ordered structure. Experimentally, it is known that the structured arrays of microdroplets can be observed over tea, coffee, tap water, distilled water and other kinds of liquid [7, 8]. Coalescence of a monolayer is a chain reaction: one drop touches the water layer, creating a capillary wave on the surface, and that destroys adjacent drops [9]. The process takes a split second and visually looks like an instant disappearance of the monolayer. However, using a high-speed video camera (at least 30000 frames per second), it is possible to visualize the details of this process. In the papers $[5,6,10]$, the behavior of levitating liquid microdroplets near the contact line was studied. It was found that when approaching the contact line, the droplet levitation height increases several times, which possibly indicates the existence of intense evaporation near the contact line. In addition, over the dry surface near the contact line exist a "forbidden zone", where drops are not observed. The width of the forbidden zone varies from 25 to $75 \mu \mathrm{m}$. Moreover, three scenarios of the migration of liquid microdroplets over the contact line to the dry area were revealed: 1) droplets fly through the contact line with a change in the levitation altitude, 2) the drops travel along a circular path near the contact line with one or more complete rotations and fall onto a dry surface, 3) some drops levitate at a fixed location. Levitation of drops over a heated dry surface was observed in many experimental studies on the Leidenfrost effect [13, 14], but self-organization of microdroplets into structures, as described in [2, 11, 12], was not researched before. Leidenfrost's levitation model to the case of $[2,11,12]$, is not applicable because the Leidenfrost temperature $\left(\mathrm{a} 200{ }^{\circ} \mathrm{C}\right)$ is higher than substrate temperature in $[2,11,12]$ (a 55 $\left.{ }^{\circ} \mathrm{C}\right)$. In addition, a theoretical model that satisfactorily describes the dependence of the levitation height on the droplet size was offered.

The aim of this work is to determine the size of monolayer and the distance between drops depending on the main parameters.

\section{Experimental setup}

The test section is a plate of stainless steel base in the centre of which a copper rod with size $10 \times 10 \mathrm{~mm}^{2}$ is pressed, which serves as a heating element. Thermocouples were installed to measure the temperature of the copper rod surface. The temperature of heater surface is $50-100{ }^{\circ} \mathrm{C}$. The entire experimental setup was placed in a box that minimized the external airflows. The test section is oriented horizontally and open to the atmosphere. The ambient temperature is $20-25^{\circ} \mathrm{C}$. As a working fluid ultra-pure, nanofiltered water Milli-Q is used. The initial temperature of working liquid is $25 \pm 1{ }^{\circ} \mathrm{C}$. The specified volume of the working fluid is applied by means of a syringe to the working surface with the formation of a liquid film. The thickness of the film is calculated by the volume of the liquid to be filled. The film thickness varied from 0.3 to $0.9 \mathrm{~mm}$. The optical system consisting of lenses, semi-transparent mirror and digital camera was used. The optical resolurion $-10 \mu \mathrm{m} / \mathrm{pix}$. The scheme of the experimental setup is shown in Fig. 2. 


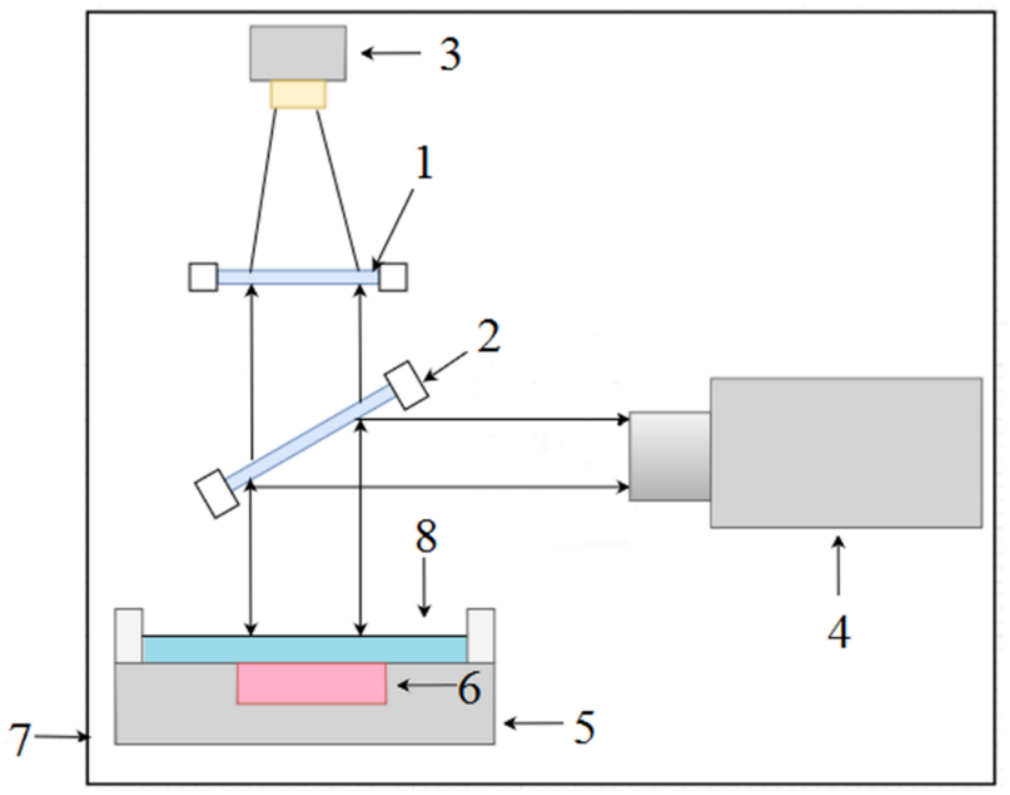

Fig. 2. The scheme of the experimental setup: 1-lens, 2- semi-transparent mirror, 3- light source, 4camera, 5-stainless steel plate, 6-heater, 7-box.

\section{Experimental results}

In most cases, the monolayer had an elliptical shape (Fig. 1). Fig. 3. shows the dependence of average diameter on time.

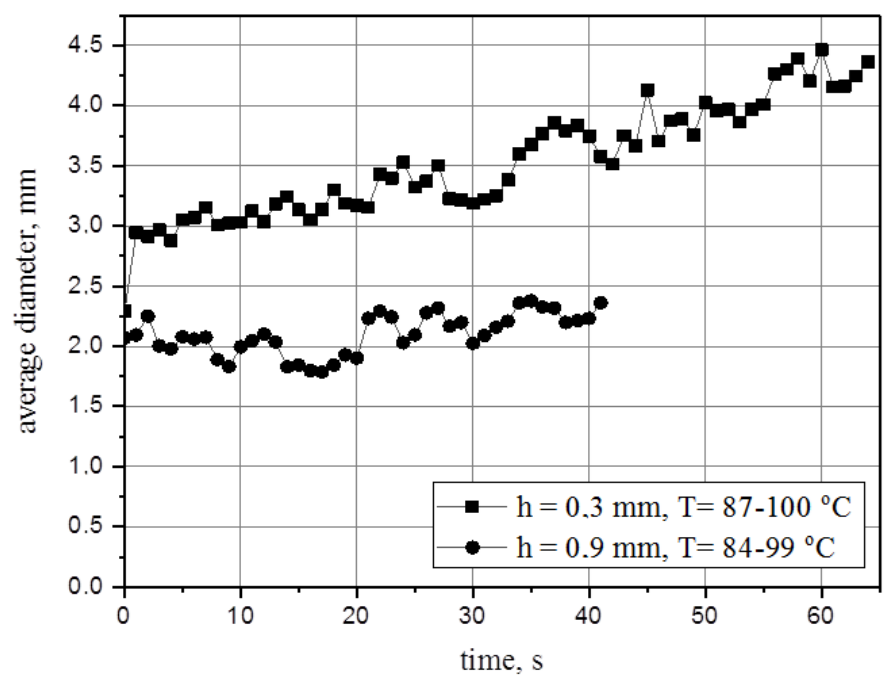

Fig. 3. The average diameter vs. time. 


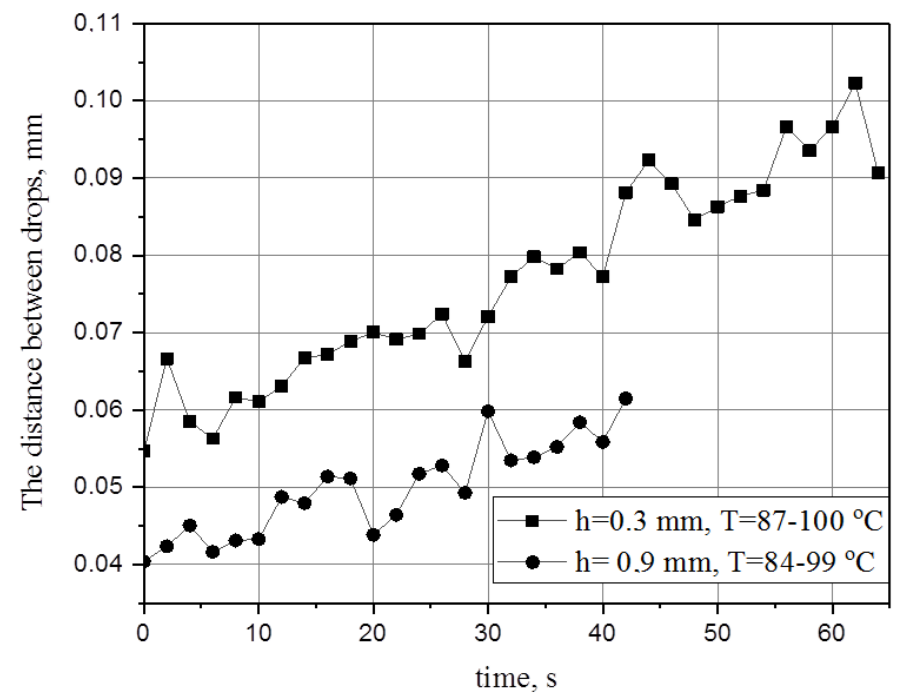

Fig. 4. The distance between drops vs. time.

The average diameter is defined as sum of semi-minor axis and semi-major axis. The dependence was obtained for two different monolayers with varying thickness of liquid layer $(\mathrm{h}=0.3$ or $0.9 \mathrm{~mm})$ and temperature $\left(\mathrm{T}=84-100^{\circ} \mathrm{C}\right)$. As can be seen from Fig. 3 the average diameter increases with decreasing thickness of the liquid layer. The average diameter increases with time from 1 to $5 \mathrm{~mm}$. Fig. 4 shows the dependence of the distance between drops on time. The interdroplet distance increases with decreasing the thickness of the liquid film.

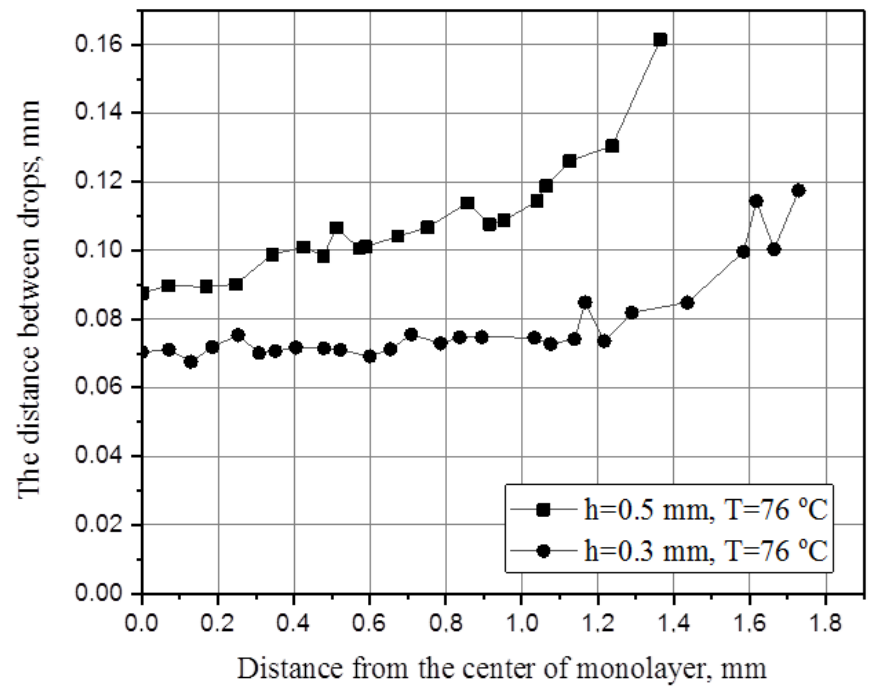

Fig. 5. The distance between drops vs. The distance from the center of monolayer.

The distance between drops varies from 0.04 to $0.1 \mathrm{~mm}$. The lines in Figure 4 correspond to the same two monolayers as in Figure 3. Figure 5 shows a plot of the distance between the drops depending on the distance of the center of monolayer. The dependence is presented for two different monolayers at different thicknesses of the liquid layer $(\mathrm{h}=0.3$ or $\mathrm{h}=0.5 \mathrm{~mm})$ but at the same temperatures $\left(\mathrm{T}=76^{\circ} \mathrm{C}\right)$. The peculiarity of this graph is 
that the processing was carried out using one image only, i.e. all parameters are the same. As can be seen from the Fig. 5. the distance between the drops increases when approach the boundary of monolayer.

\section{Summary}

The monolayer parameters such as the size of monolayer, the distance between the drops depends on many parameters such as temperature, the thickness of liquid layer. The average diameter and interdroplet distance increases with decreasing the thickness of the liquid layer. The average diameter of monolayer increase from 1 to $5 \mathrm{~mm}$. Moreover, the distance between drops varies from 40 to $100 \mu \mathrm{m}$. The interdroplet distance increase when approaching the periphery of the monolayer.

The research was supported by the Russian Science Foundation, Project No. 1419-01755.

\section{References}

1. A. Fedorets JETP Lett. 79, 372 (2004)

2. D. V. Zaitsev, D. P. Kirichenko, V. S. Ajaev, O. A. Kabov, Phys. Rev. Lett. 19, 094503 (2017)

3. J. Kim, Int. J. Heat Fluid Flow 28, 753 (2007)

4. Z. Zhang, C. Kleinstreuer, J. Donohue, C. Kim, Aerosol Sci. 36, 211 (2005)

5. O. A. Kabov, D. V. Zaitsev, D. P. Kirichenko, V. S. Ajaev, Nanosc. Microsc. Thermophys. Eng. 21, 60 (2017)

6. O. A. Kabov, D. V. Zaitsev, D. P. Kirichenko, V. S. Ajaev, Interfacial Phenomena and Heat Transfer 4, 207 (2016)

7. T. Umeki, M. Ohata, H. Nakanishi, M. Ichikawa, Sci. Rep. 58046 (2015)

8. V. J. Schaefer Am. Sci. 59, 534 (1971)

9. A. Fedorets, I. Marchuk, P. Stryzhak, O. Kabov, Thermophys. Aeromechanics 22, 535 (2015)

10. D. V. Zaitsev, D. P. Kirichenko, O. A. Kabov, MATEC Web Conf. 92, 01026 (2017)

11. D. V. Zaitsev, D. P. Kirichenko, O. A. Kabov, MATEC Web Conf. 84, 00044 (2016)

12. D. P. Kirichenko, D. V. Zaitsev, O. A. Kabov, J. Phys. Conf. Ser. 754, 042008 (2016)

13. D. Quere, Annu. Rev. Fluid Mech. 45, 197 (2013)

14. F. Celestini, T. Frisch, Y. Pomeau, Phys. Rev. Lett. 109, 034501 (2012) 